\title{
Vibrational Stark Fields in Carboxylic Acid Dimers
}

\author{
Manjusha Boda, and G. Naresh Patwari* \\ Department of Chemistry, Indian Institute of Technology Bombay, Powai Mumbai 400076, \\ India \\ E-mail: naresh@chem.iitb.ac.in
}

\begin{abstract}
Carboxylic acids form exceptionally stable dimers and have been used to model proton and double proton transfer processes. The stabilization energies of the carboxylic acid dimers are very weakly dependent on the nature of the substitution. However, the electric field experienced by the $\mathrm{OH}$ group of a particular carboxylic acid is dependent more on the nature of the substitution on the dimer partner. In general, the electric field was higher when the partner was substituted with electron-donating group and lower with electron-withdrawing substituent on the partner. The Stark tuning rate $(\Delta \vec{\mu})$ of the $\mathrm{O}-\mathrm{H}$ stretching vibrations calculated at the MP2/aug-cc-pVDZ level was found to be weakly dependent on the nature of substitution on the carboxylic acid. The average Stark tuning rate of $\mathrm{O}-\mathrm{H}$ stretching vibrations of a particular carboxylic acid when paired with other acids was $5.7 \mathrm{~cm}^{-1}\left(\mathrm{MV} \mathrm{cm}^{-1}\right)^{-1}$, while the corresponding average Stark tuning rate of the partner acids due to a particular carboxylic acid was $21.9 \mathrm{~cm}^{-1}$ (MV $\left.\mathrm{cm}^{-1}\right)^{-1}$. The difference in the Stark tuning rate is

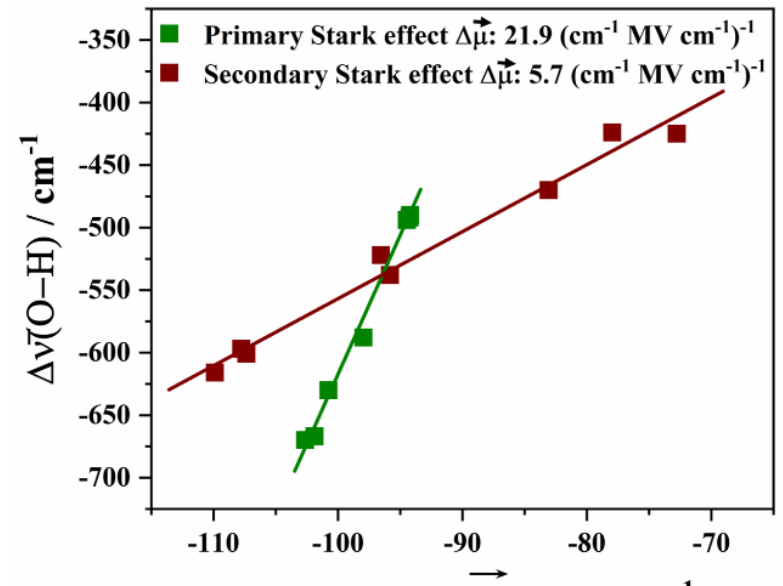

Electric Field $\overrightarrow{(\mathbf{E})} / \mathrm{MV} \mathrm{cm}^{-1}$ attributed to the primary and secondary effects of substitution on the carboxylic acid. The average Stark tuning rate for the anharmonic 0-D frequency shifts is about 40-50\% higher than the corresponding harmonic O-D frequency shifts calculated at B3LYP/aug-cc-pVDZ level, much greater than the typical scaling factors used, indicating the strong anharmonicity of $\mathrm{O}-\mathrm{H} / \mathrm{O}-\mathrm{D}$ oscillators in carboxylic acid dimers. Finally, the linear correlation observed between $p K_{a}$ and the electric field was used to estimate the $p \mathrm{~K}_{\mathrm{a}}$ of fluoroformic acid to be around 0.9.
\end{abstract}




\section{Introduction}

Carboxylic acids are well-known to form doubly hydrogen-bonded cyclic dimers with exceptional stability, wherein each monomer acts both as a donor and acceptor, and have been used as supramolecular synthons. ${ }^{1}$ These dimers are readily identified by their characteristic sharp band in the $1680-1725 \mathrm{~cm}^{-1}$ region and a broadband in the $2500-3000 \mathrm{~cm}^{-1}$ region corresponding to $\mathrm{C}=\mathrm{O}$ and $\mathrm{O}-\mathrm{H}$ stretching bands, respectively. ${ }^{2,3}$ The IR spectra of carboxylic acid dimers, especially in the $\mathrm{O}-\mathrm{H}$ stretching region, have been a subject of interest for a long time since these spectra carry the most significant information about the structure and dynamics of the dimer. However, the interpretation of the IR spectra, especially in the $\mathrm{O}-\mathrm{H}$ stretching region has been difficult due to vibrational coupling, tunneling-splitting, and anharmonicity. ${ }^{4-11}$ One of the interesting models to interpret the IR spectra in the $\mathrm{O}-\mathrm{H}$ stretching region of carboxylic acid dimers is monomers in the dimer model. ${ }^{12}$ However, the role of hydrogen bonding in the interpretation of these spectra has been reduced to tuning the $\mathrm{OH}$ stretching vibration in and out of resonance with combinations of the remaining vibrational modes of the $\mathrm{COOH}$ functional group. The carboxylic acid dimers, especially the formic acid dimer, have been used as a model system to understand the proton transfer including double proton transfer processes, ${ }^{13-17}$ and proton tunneling. ${ }^{18}$ Various substitutions are known to affect the stability of carboxylic acid dimers, which can be modulated by substituting electrondonating or electron-withdrawing groups.

The importance of high stability of carboxylic acids dimers has been highlighted in various aspects including their ability to dissociate in an appropriate solvent, ${ }^{19-23}$ and the dissociation of carboxylic acids in any solvent is a competitive process that involves breaking of the doubly hydrogen-bonded dimer in the presence of the solvent and the interaction with the solvent leading to acid dissociation. ${ }^{20}$ Based on such models, the $p \mathrm{~K}_{\mathrm{a}}$ of several carboxylic acids have been estimated using a combination of electronic structure calculations with solvation modes and thermodynamic cycles with reasonable accuracy. ${ }^{24-27}$ The presence of electron-donating substituents such as alkyl groups $\left[\mathrm{R}=\mathrm{CH}_{3}-, \mathrm{CH}_{3} \mathrm{CH}_{2}-,\left(\mathrm{CH}_{3}\right)_{2} \mathrm{CH}-\right]$ lowers the ability to deprotonate, whereas the presence of electron-withdrawing substituents such fluorine and fluoroalkyl groups $\left[\mathrm{R}=\mathrm{F}-, \mathrm{CH}_{2} \mathrm{~F}-, \mathrm{CHF}_{2}-, \mathrm{CF}_{3}-\right]$ increases the ability to dissociate the carboxylic acid. The carboxylic acids are known to form highly stable dimers, 


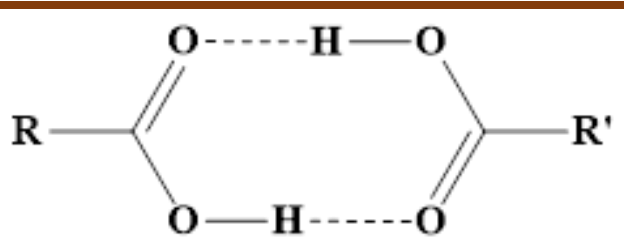

Fig. 1 Carboxylic acid dimers investigated in the present work. $\mathrm{R}, \mathrm{R}^{\prime}=\mathrm{H}, \mathrm{CH}_{3}, \mathrm{CH}_{3} \mathrm{CH}_{2}$, $\left(\mathrm{CH}_{3}\right)_{2} \mathrm{CH}, \mathrm{F}, \mathrm{CH}_{2} \mathrm{~F}, \mathrm{CHF}_{2}$ and $\mathrm{CF}_{3}$.

the stabilization energy is related to the entire dimer which includes the electrostatic and dispersion effects. However, to understand the role of the $\mathrm{OH}$ group in the dimer, the electric field along the $\mathrm{O}-\mathrm{H}$ bond is an appropriate descriptor as it captures the local environment, 28,29 which can also be estimated experimentally. ${ }^{30}$ In the present work, the electric fields experienced along the $\mathrm{OH}$ group in carboxylic acid dimers were calculated and correlated with the frequency shifts in the hydrogen-bonded $\mathrm{OH}$ groups for several (eight) different series of carboxylic acid dimers. A schematic representation of carboxylic acid dimer is shown in Fig. 1 with $\mathrm{R}$ and $\mathrm{R}^{\prime}$ substituents on the acid monomeric unit and partner monomeric unit respectively. In an effort to understand the role of local internal electric fields on the structure and $\mathrm{O}-\mathrm{H}$ stretching vibrations, eight carboxylic acid monomeric units, namely, formic acid (FA, $\mathrm{R}=\mathrm{H}$ ), acetic acid ( $\mathrm{AA}, \mathrm{R}=\mathrm{CH}_{3}$ ), propanoic acid ( $\mathrm{PA}, \mathrm{R}=\mathrm{CH}_{3} \mathrm{CH}_{2}$ ), methylpropanoic acid (MPA, $\mathrm{R}=$, $\left(\mathrm{CH}_{3}\right)_{2} \mathrm{CH}$ ), fluoroformic acid (FFA, $\mathrm{R}=\mathrm{F}$ ), fluoroacetic acid (FAA, $\mathrm{R}=\mathrm{CH}_{2} \mathrm{~F}$ ), difluoroacetic acid (DFAA, $\mathrm{R}=\mathrm{CHF}_{2}$ ) and trifluoroacetic acid (TFAA, $\mathrm{R}=\mathrm{CF} 3$ ) were considered.

\section{Methodology}

The structure of several carboxylic acid monomers and dimers (both homo and hetero) were optimized using MP2 and B3LYP-D3 methodologies with aug-cc-pVDZ basis set and frequency calculations were carried out using the same level of theory. The stabilization energies of the clusters were evaluated as the difference in energy of the dimer and sum of the energies of the corresponding monomers and were corrected for the vibrational zero-point energy (ZPE). The two $\mathrm{OH}$ oscillators in a carboxylic acid dimer are strongly coupled and these two vibrations are more aptly described as symmetric and antisymmetric vibrations of the dimer. Additionally, the $\mathrm{OH}$ oscillators are also coupled to 
the $\mathrm{CH}$ oscillators of the alkyl group in some cases. Therefore, two strategies were adopted to uncouple the $\mathrm{OH}$ oscillators in the dimer. In the first method, one of the $\mathrm{OH}$ groups was kept intact and the rest of the hydrogen atoms in the dimer were substituted with deuterium. In the second method, one of the $\mathrm{OH}$ groups was replaced with the OD group and the rest of the hydrogen atoms were kept intact. In the first case, the resulting uncoupled $\mathrm{O}-\mathrm{H}$ and in the second case uncoupled $\mathrm{O}-\mathrm{D}$ stretching vibrations were analyzed, relative to the $\mathrm{O}-\mathrm{H}$ and $\mathrm{O}-\mathrm{D}$ stretching vibrations of the corresponding monomer. Further, anharmonic vibrational frequencies were also calculated using the second-order vibrational perturbation theory (VPT2) using the B3LYP-D3/aug-cc-pVDZ level of theory implemented in Gaussian-16. In order to calculate the electric field along each of the hydrogen-bonded $\mathrm{OH}$ group, ${ }^{28,31,32}$ the molecular electrostatic potential (MESP) on the position of the $\mathrm{O}$ and $\mathrm{H}$ atoms of one of the carboxylic acid due to its partner carboxylic acid was calculated by replacing the relevant $\mathrm{O}$ and $\mathrm{H}$ atoms with ghost atoms and removing the rest of the atoms that constitute the molecule of which the $\mathrm{OH}$ group was part. The MESP on the atom $\mathrm{H} / \mathrm{O}$ located at the position $R_{H} / R_{O}$ is given by equation $(1)^{33}$

$$
V_{O / H}=\sum_{A \neq O, H} \frac{Z_{A}}{\left|\vec{R}_{A}-\vec{R}_{O / H}\right|}-\int \frac{\rho(\vec{r}) d^{3} \vec{r}}{\left|\vec{r}-\vec{R}_{O / H}\right|}
$$

where $Z_{\mathrm{A}}$ is the nuclear charge of atom A located at $R_{\mathrm{A}}, \rho(\mathrm{r})$ is the electron density of the molecule and $r$ is a dummy integration variable. The projection of the electric field onto the bond was calculated as the gradient of the electrostatic potential along the bond axis along the $\mathrm{O}-\mathrm{H}$ direction, which is given by equation (2).

$$
\vec{E}=-\nabla V=\frac{-\left(V_{O}-V_{H}\right)}{\left|\vec{R}_{O}-\vec{R}_{H}\right|}
$$

in equation (2) $V_{o}$ and $V_{\mathrm{H}}$ are the measures of the electrostatic potential at the position of atoms $\mathrm{O}$ and $\mathrm{H}$ due to all the electrons and nuclei of the partner carboxylic acid. All the calculations were carried out using the Gaussian-16 suite of programs. ${ }^{34}$ 


\section{Results and Discussion}

A total of thirty-six possible carboxylic acid dimers, i.e., eight homodimers and twenty-eight heterodimers formed from the chosen eight carboxylic acid monomers were considered in the present work and the dimerization energies are listed in Tables S1 and S2 (see the Supporting Information, SI). The energies for the dimerization of all the thirty-six pairs lie in the range of 60.2-71.5 (and 60.9-70.0) kJ mol-1 with an average value of 65.7 (and 63.7) kJ mol-1 for the MP2 (and B3LYP-D3) levels of theory, respectively. Further, the electric field was calculated along each of the hydrogen-bonded $\mathrm{OH}$ groups and the total electric field in each dimer (sum of electric field on two OH groups in the dimer) is also listed in Table S1 and S2 at MP2 and B3LYP-D3 levels of theory (see the SI), respectively. In general, the stabilization energies calculated at the MP2 and B3LYP-D3 level differ only marginally, while the magnitude of electric fields values calculated at the MP2 level are about $15 \mathrm{MV} \mathrm{cm}^{-1}$ higher.

As noted earlier, the $\mathrm{OH}$ stretching vibrations of carboxylic acid dimers have been the subject of many investigations. In the present work, the shifts in the $\mathrm{OH}$ stretching vibrations due to dimerization were analyzed in terms of the Stark field along the uncoupled $\mathrm{OH}$ groups. Table S3 and S4 (see the SI) lists all the $\mathrm{O}-\mathrm{H}$ and $\mathrm{O}-\mathrm{D}$ and stretching frequencies and the corresponding electric fields calculated in the MP2 level of theory, respectively. The electric fields for a various carboxylic acid dimers vary in the range - 155 to $-216 \mathrm{MV} \mathrm{cm}^{-1}$ and are in the same range as water and methanol clusters. ${ }^{28,35}$ The plots of red-shifts in the $\mathrm{O}-\mathrm{H}$ and $\mathrm{O}-\mathrm{D}$ stretching frequencies as a function of the corresponding electric field for the dimers of acetic acid (AA) and trifluoroacetic acid (TFAA) calculated at MP2/aug-cc-pVDZ level of theory are shown in Fig. 2. Similar data plots for the remaining six carboxylic acids (viz., FA, PA, MPA, FFA, FAA, and DFAA are shown in Fig. S1, see the SI). The shifts in the O-H/O-D stretching frequencies of carboxylic acid dimers are categorized into two sets. First, the red-shift in the O$\mathrm{H}$ and O-D stretching frequency of a particular carboxylic acid (acetic acid/trifluoroacetic acid in Fig. 2) due to dimerization with other carboxylic acids, shown as solid squares and solid circles respectively in Fig. 2. Second, the red-shift in the $\mathrm{O}-\mathrm{H}$ and $\mathrm{O}-\mathrm{D}$ stretching frequencies of

all the carboxylic acids due to dimerization with a particular carboxylic acid (acetic acid/trifluoroacetic acid in Fig. 2), shown as open squares and open circles respectively in Fig. 
2. In all the cases the data could be fitted to straight lines and the slope of the linear fits yields Stark tuning rate which is given by equation (3)

$$
\Delta \bar{v}=\Delta \vec{\mu} \cdot \vec{E}
$$

It is interesting to note from Fig. 2 that for a particular carboxylic acid, two sets of data points were observed. For instance, in Fig. 2A the solid squares correspond to the shifts in the $\mathrm{O}-\mathrm{H}$ stretching frequency of acetic acid when partnered (dimerized) with other carboxylic acids and a linear fit to these data points yields a Stark tuning rate of $4.9 \mathrm{~cm}^{-1}[\mathrm{MV} \mathrm{cm}-1]^{-1}$. On the other hand, open squares represent a shift in the $\mathrm{O}-\mathrm{H}$ stretching frequencies of all the carboxylic acids partnered with acetic acid, and linear fit to these data points yields a Stark tuning rate $(\Delta \vec{\mu})$ of $24.1 \mathrm{~cm}^{-1}\left[\mathrm{MV} \mathrm{cm}^{-1}\right]^{-1}$. Similarly, in the case of trifluoroacetic acid (Fig. 2B) the Stark tuning rate $(\Delta \vec{\mu})$ for the $\mathrm{O}-\mathrm{H}$ group of trifluoroacetic acid upon partnering with other carboxylic acids is $6.9 \mathrm{~cm}^{-1}\left[\mathrm{MV} \mathrm{cm}^{-1}\right]^{-1}$, while the effect of trifluoroacetic acid on the $0-\mathrm{H}$ group of its partners yields a Stark tuning rate $(\Delta \vec{\mu})$ of $19.5 \mathrm{~cm}^{-1}\left[\mathrm{MV} \mathrm{cm}^{-1}\right]^{-1}$. Table 1 lists the Stark tuning rates $(\Delta \vec{\mu})$ for all the eight sets of carboxylic acid dimers considered in the present work, while the fitting parameters (residuals and correlation coefficients) are listed in Tables S7 and S8 (see the SI). In comparison, the stark tuning rates for the water and methanol clusters were 2.4 and 3.1 [MV $\left.\mathrm{cm}^{-1}\right]^{-1}$, respectively. The Stark tuning rate is the change in the dipole moment of the $0-\mathrm{H} / 0-\mathrm{D}$ due to the electric field and is a measure of the efficacy of a vibrational probe to hydrogen bonding, 30 therefore the $\mathrm{OH}$ group of carboxylic acids has higher sensitivity as a vibrational probe relative to the $\mathrm{OH}$ group of water and methanol.28,35 It must be noted that the electric field values in the present work are absolute, unlike in the case of hydrogen-bonded $\mathrm{OH}$ group of phenol, wherein the electric field values are relative to the $\mathrm{CCl}_{4}$ as solvent. ${ }^{32}$ However, unlike phenolic $\mathrm{OH}$ group exhibiting non-linear vibrational stark effect on the at larger fields, in the present case only linear response is observed, similar to water and methanol clusters. ${ }^{28,35}$ This difference in the non-linear/linear behaviour can be attributed the solution-phase measurement for the phenolic $\mathrm{OH}$ group ${ }^{32}$ and the calculation being carried out for gas-phase clusters.

In order to estimate the confidence limit of the present set of calculations, dimers of formic acid (FA) were recalculated at MP2/aug-cc-pVTZ level and the plot of the red-shifts in the $\mathrm{O}-\mathrm{H}$ stretching frequencies against the corresponding Stark fields for FA dimers are shown 

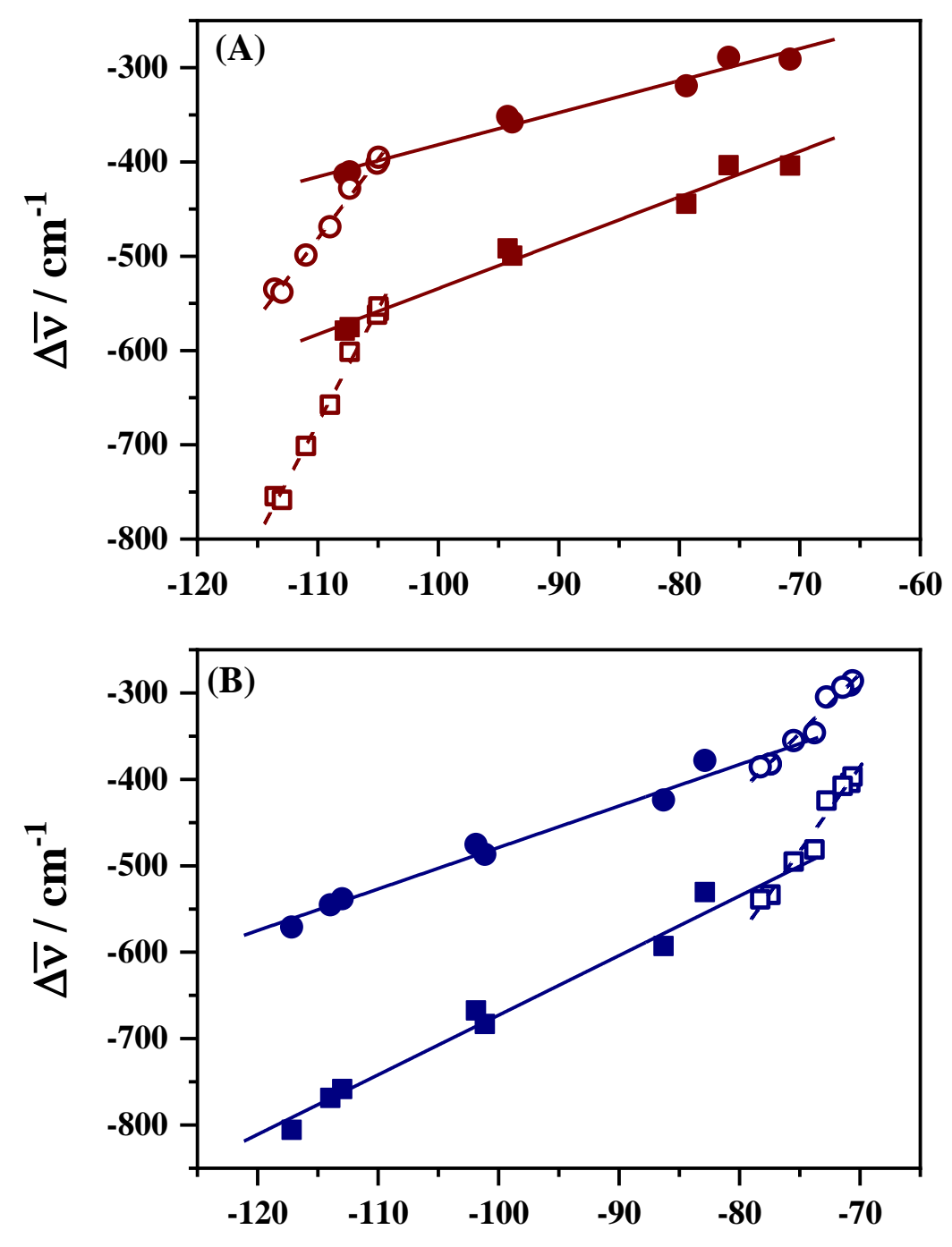

Electric Field $(\overrightarrow{\mathbf{E}}) / \mathrm{MV} \mathrm{cm} \mathbf{c m}^{-1}$

Fig. 2 Plots of the red-shifts in the $\mathrm{O}-\mathrm{H}$ (squares) and O-D (circles) stretching frequencies against the corresponding Stark fields in the dimers of (A) acetic acid and (B) trifluoroacetic acid calculated at MP2/aug-cc-pVDZ level of theory. The solid symbols represent the shifts in the $\mathrm{O}-\mathrm{H} / \mathrm{O}-\mathrm{D}$ stretching frequencies of acetic acid/trifluoroacetic acid due to change in the substituent on the dimerizing partner, while open symbols represent the shifts in the $\mathrm{O}-\mathrm{H} / \mathrm{O}-\mathrm{D}$ stretching frequencies of the other carboxylic acids due to their dimerization with acetic acid/trifluoroacetic acid. The straight-lines are linear least-square fits to the data points and the slopes of these straight lines are called Stark tuning rates $(\Delta \vec{\mu})$. 
Table 1: Stark tuning rates $\Delta \vec{\mu}\left(\mathrm{cm}^{-1}\left[\mathrm{MV} \mathrm{cm}{ }^{-1}\right]^{-1}\right)$ for the eight sets of carboxylic acid hetero dimers calculated for the $0-\mathrm{H}$ and 0 -D stretching frequencies at various levels of theory using aug-cc-pVDZ basis set. The column $\Delta \vec{\mu}(\mathrm{R})$ corresponds to Stark tuning rates for a carboxylic acid due to change in the substituent of the dimerization partner, while the column $\Delta \vec{\mu}\left(R^{\prime}\right)$ corresponds to Stark tuning rates for all the carboxylic acids partnered with a given carboxylic acid.

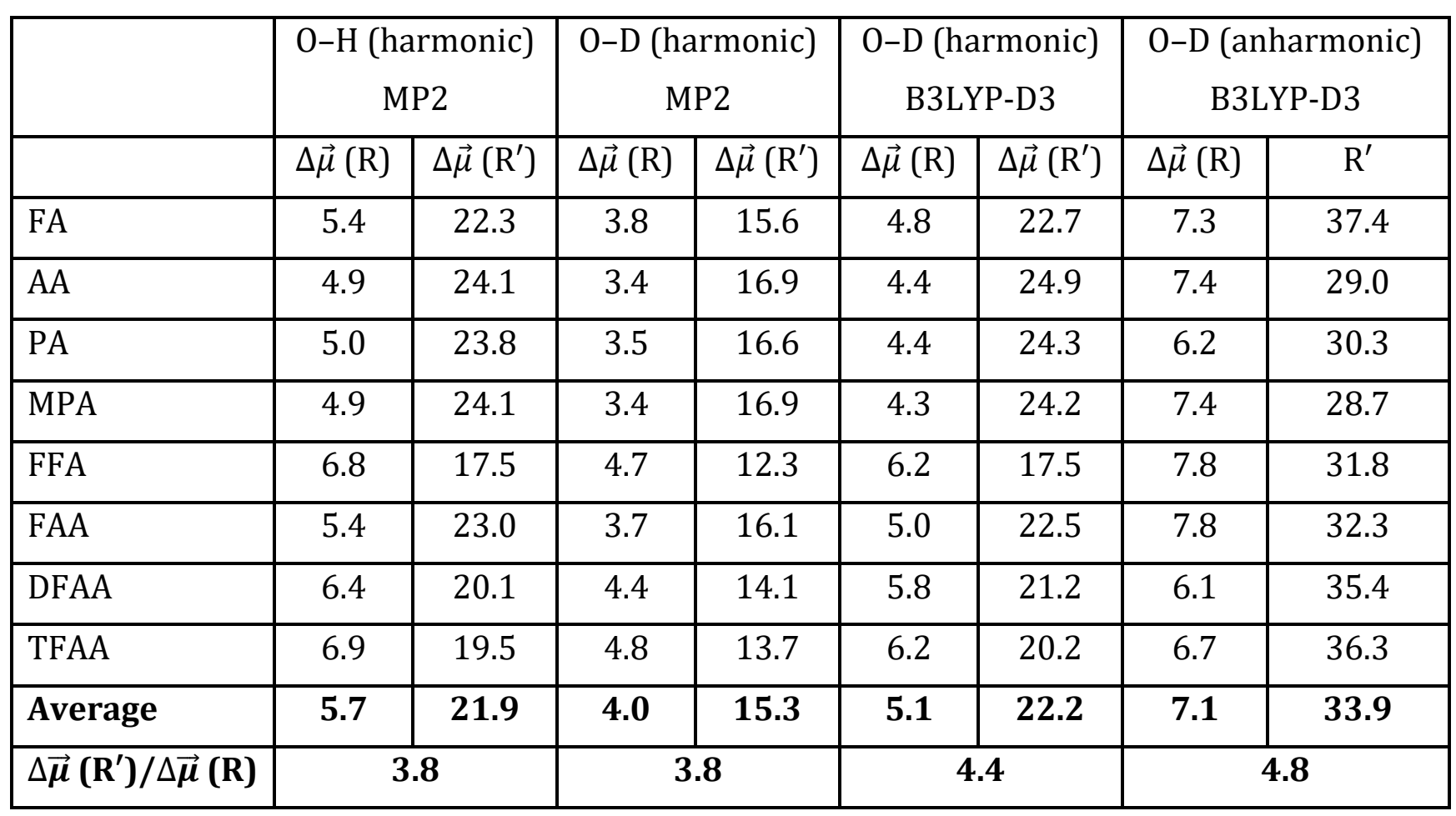

in Fig. S2 (see the SI) along with those calculated at the MP2/aug-cc-pVDZ level for the sake of comparison. Even though the absolute values of the frequency shifts are about $30-50 \mathrm{~cm}^{-1}$ larger at the MP2/aug-cc-pVTZ level, the stark tuning rates $(\Delta \vec{\mu})$ are however are very comparable (5.4 vs 5.5 and 22.3 vs 24.2 ) and are very weakly dependent on the basis set (aug-cc-pVDZ vs. augcc-pVTZ). In Table 1 , the column $\Delta \vec{\mu}(\mathrm{R})$ and $\Delta \vec{\mu}\left(\mathrm{R}^{\prime}\right)$ correspond to the Stark running rate $(\Delta \vec{\mu})$ values for a carboxylic acid with change in the substituent of the partner in the dimer, and for all the carboxylic acid dimers partnered with a carboxylic acid. It is well known that the anharmonicity is considerably important in the $\mathrm{O}-\mathrm{H}$ stretching vibration of the carboxylic acid dimer as they form a double-well potential and is addressed in several literature reports. The role of anharmonicity on O-D frequency shifts and consequent Stark tuning rate was estimated by calculating the $0-\mathrm{D}$ frequencies of the individual carboxylic acids in the dimer using the 

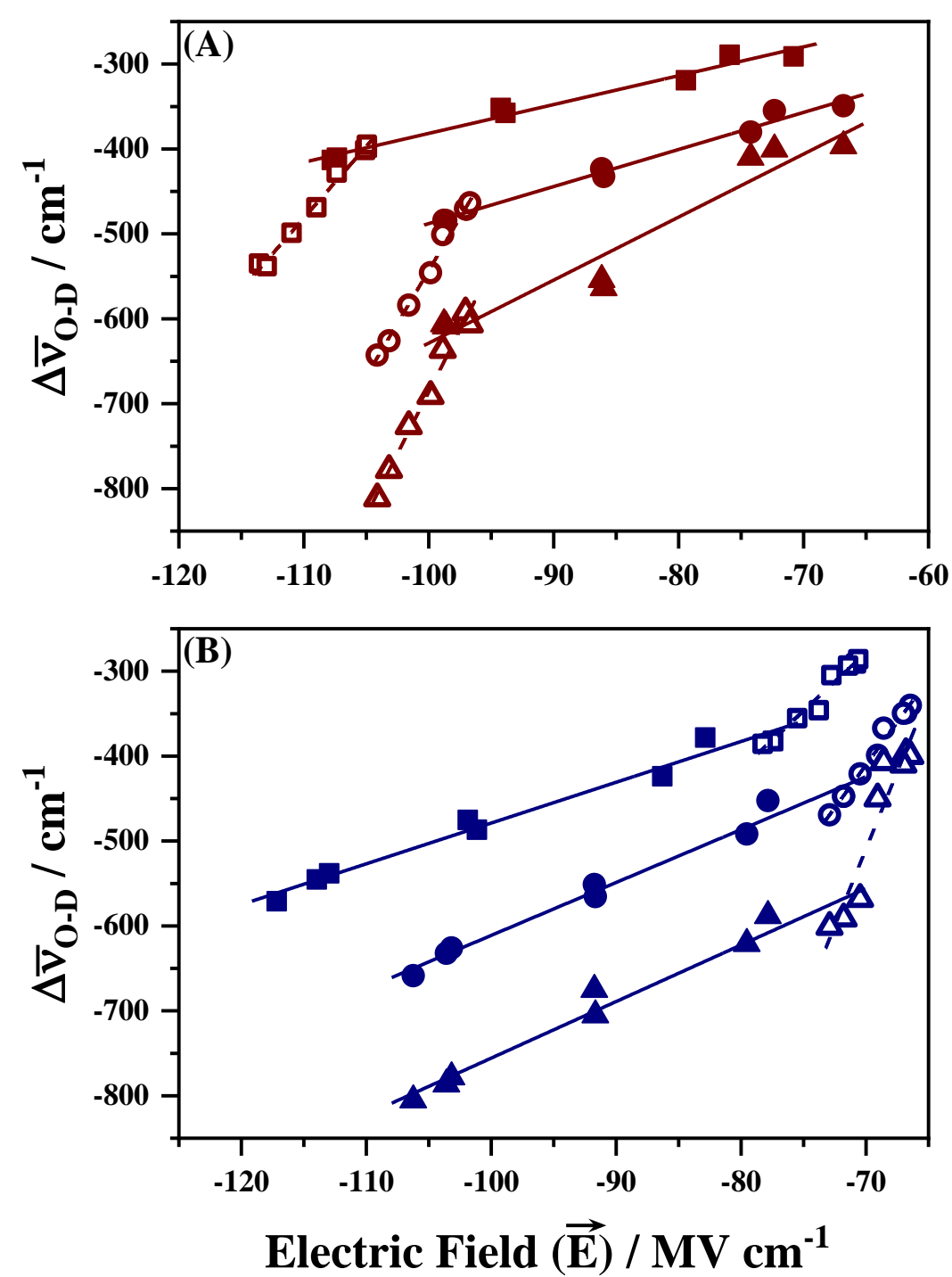

Fig. 3 Plots of the red-shifts in the O-D stretching frequencies against the corresponding Stark fields in the dimers of (A) acetic acid and (B) trifluoroacetic acid. In each case, the squares, circles, and triangles represent harmonic MP2, harmonic B3LYP-D3, and anharmonic B3LYPD3frequency shifts calculated using aug-cc-pVDZ basis set, respectively. Additionally, the solid symbols represent the change in the $\mathrm{O}-\mathrm{D}$ stretching frequencies of the acetic acid/trifluoroacetic $(R)$ with change in the partner $\left(R^{\prime}\right)$. On the other hand, the open symbols represent the change in the $0-D$ stretching frequencies of partner $\left(R^{\prime}\right)$ when dimerized with acetic acid /trifluoroacetic acid. The straight-lines are linear least-square fits to the data points.

VPT2 method using B3LYP-D3/aug-cc-pVDZ level of theory. This calculation was repeated twice in the case of hetero-dimer to account for both the OH groups. Fig. 3 shows the plot of shifts in the O-D stretching frequencies of acetic acid (panel A) and trifluoroacetic acid (panel B) 
calculated using MP2 (harmonic), B3LYP-D3 (harmonic) and B3LYP (anharmonic) level of theories using aug-cc-pVDZ basis set against the corresponding electric field. Similar to Fig. 2, two sets of correlations, first the effect of dimerizing partner on a given carboxylic acid (column $\Delta \vec{\mu}(\mathrm{R})$ in Table 1) and second the effect of a given carboxylic acid on the dimerizing partner (column $\Delta \vec{\mu}\left(\mathrm{R}^{\prime}\right)$ in Table 1), were observed. The plots for the remaining six data sets are shown in Fig. S3 (see the SI). Based on the data presented in Figs 2, 3, S1 and S3 along with Table 1, the following observations can be made.

(i) The Stark tuning rates $(\Delta \vec{\mu})$ for the 0 -D stretching vibrations are lower by about a factor of $\sqrt{2}$ lower than the corresponding value for the $\mathrm{O}-\mathrm{H}$ stretching vibrations which can be attributed to the kinematic effect and indicates that the $\mathrm{O}-\mathrm{H} / \mathrm{O}-\mathrm{D}$ stretching vibrations are uncoupled.

(ii) For the 0-D stretching vibrations, the Stark tuning rates $(\Delta \vec{\mu})$ are in the following order: MP2 (harmonic) < B3LYP-D3 (harmonic) < B3LYP-D3 (anharmonic).

(iii) The average Stark tuning rate $(\Delta \vec{\mu})$ for the anharmonic $0-D$ frequency shifts is about 40 $50 \%$ higher than the corresponding harmonic $\mathrm{O}-\mathrm{D}$ frequency shifts, which is much greater than the typical scaling factors used, indicating the strong anharmonicity of $\mathrm{O}-\mathrm{H} / \mathrm{O}-\mathrm{D}$ oscillators in carboxylic acid dimers.

(iv) The average Stark tuning rate for a given carboxylic acid due to its variation in the substituted of the partner (column $\Delta \vec{\mu}(\mathrm{R})$ in Table 1 ) is considerably lower (factor of about 3.8-4.8) than Stark tuning rate of all the carboxylic acids partnered with a given carboxylic acid (column $\Delta \vec{\mu}\left(\mathrm{R}^{\prime}\right)$ in Table 1). See the ratio $\Delta \vec{\mu}\left(\mathrm{R}^{\prime}\right) / \Delta \vec{\mu}(\mathrm{R})$ in Table 1 .

The Stark tuning rate $\Delta \vec{\mu}(R)$ is the response of a particular carboxylic acid to the variation in the hydrogen bonding partner. This variation is influence due to the change in substitution on the partner, therefore a secondary effect. On the other hand, the Stark tuning rate $\Delta \vec{\mu}\left(\mathrm{R}^{\prime}\right)$ is the effect felt by different carboxylic acids due to a particular carboxylic acid as partner, therefore is a primary effect. The present observations clearly indicate that the primary Stark effect is about 3.8-4.8 times larger than the secondary Stark effect. This indicates that the vibrational stark response of an O-H/O-D group depends strongly on its identity and weakly on the identity of the partner. Further, the primary and the secondary Stark effects are akin to the 
primary and the secondary isotope effects. Additionally, it is expected that the primary Stark tuning rate to be almost invariant if the binding motif, as shown in Fig. 1, is conserved.

In order to probe the role of hydrogen bonding and the consequent vibrational Stark effect, all the carboxylic acid monomers were optimized in the presence of external electric field equivalent to electric calculated in various dimers along the $\mathrm{O}-\mathrm{H}$ bond direction and ensuing vibrational $\mathrm{O}-\mathrm{H}$ frequencies were calculated using MP2/aug-cc-pVDZ level and the results are listed in Table S8. Fig. 4 shows the plot of vibrational frequency shifts due to external electric field of same magnitude against the corresponding vibrational frequency shifts due to hydrogen-bonded dimer. In general, the shifts due to external electric field are much lower (about 18\% on the average) than in the hydrogen-bonded dimers, but are linearly correlated with the corresponding shifts due to formation of hydrogen-bonded dimers. Therefore, it can be concluded that Stark tuning rate for the hydrogen-bonded dimers of carboxylic acids is substantially higher than carboxylic acids in the presence of the external fields and can be

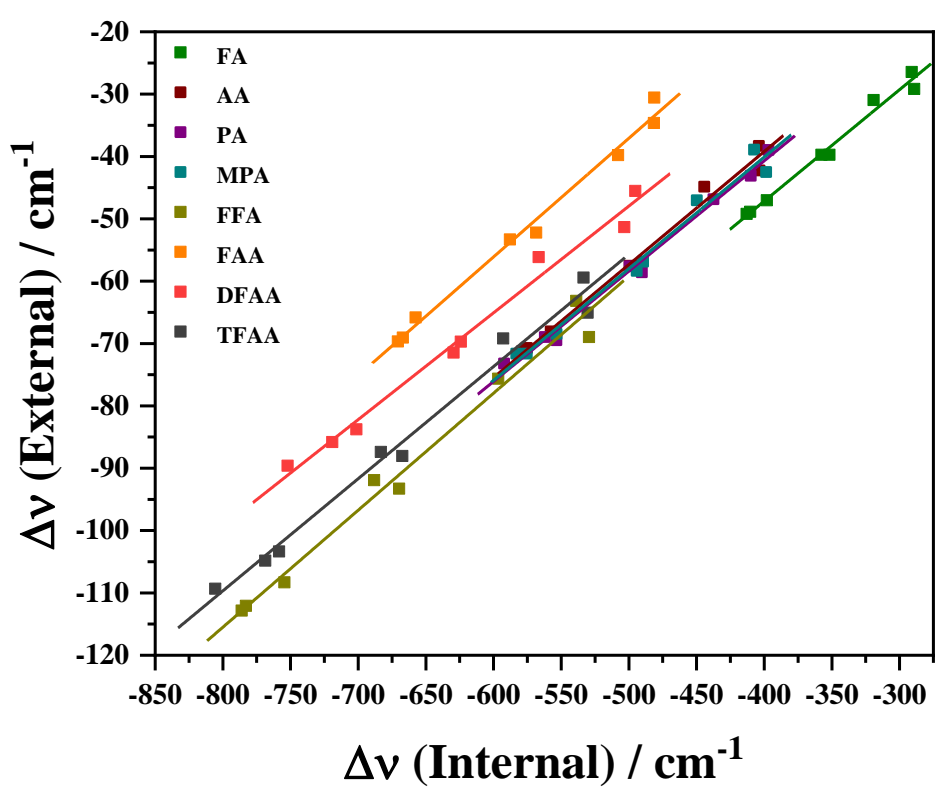

Fig. 4 Plots of the red-shifts in the $\mathrm{O}-\mathrm{H}$ stretching frequencies for various carboxylic acids presence of external electric field equivalent to the value in the hydrogen-bonded dimer against the corresponding red-shift in the hydrogen-bonded dimer. The straight lines are linear fits to the data point. The structures of the carboxylic acids were optimized using MP2/aug-cc-pVDZ level followed by frequency calculations. The average slope of the straight lines is 0.18 . 
attributed, to some extent, to donor-acceptor cooperativity in the hydrogen-bonded dimers (see Fig. 1). Qualitatively similar results differences were reported for the proton transfer reactions with the absence and presence of solvent molecules in $\mathrm{HCl}-\left[\mathrm{H}_{2} \mathrm{O}\right]_{n}$ and $\mathrm{HBr}-\left[\mathrm{H}_{2} \mathrm{O}\right]_{n}$ and $\mathrm{PhOH}-$ $\left[\mathrm{NH}_{3}\right]_{n}$ clusters. ${ }^{29,36}$
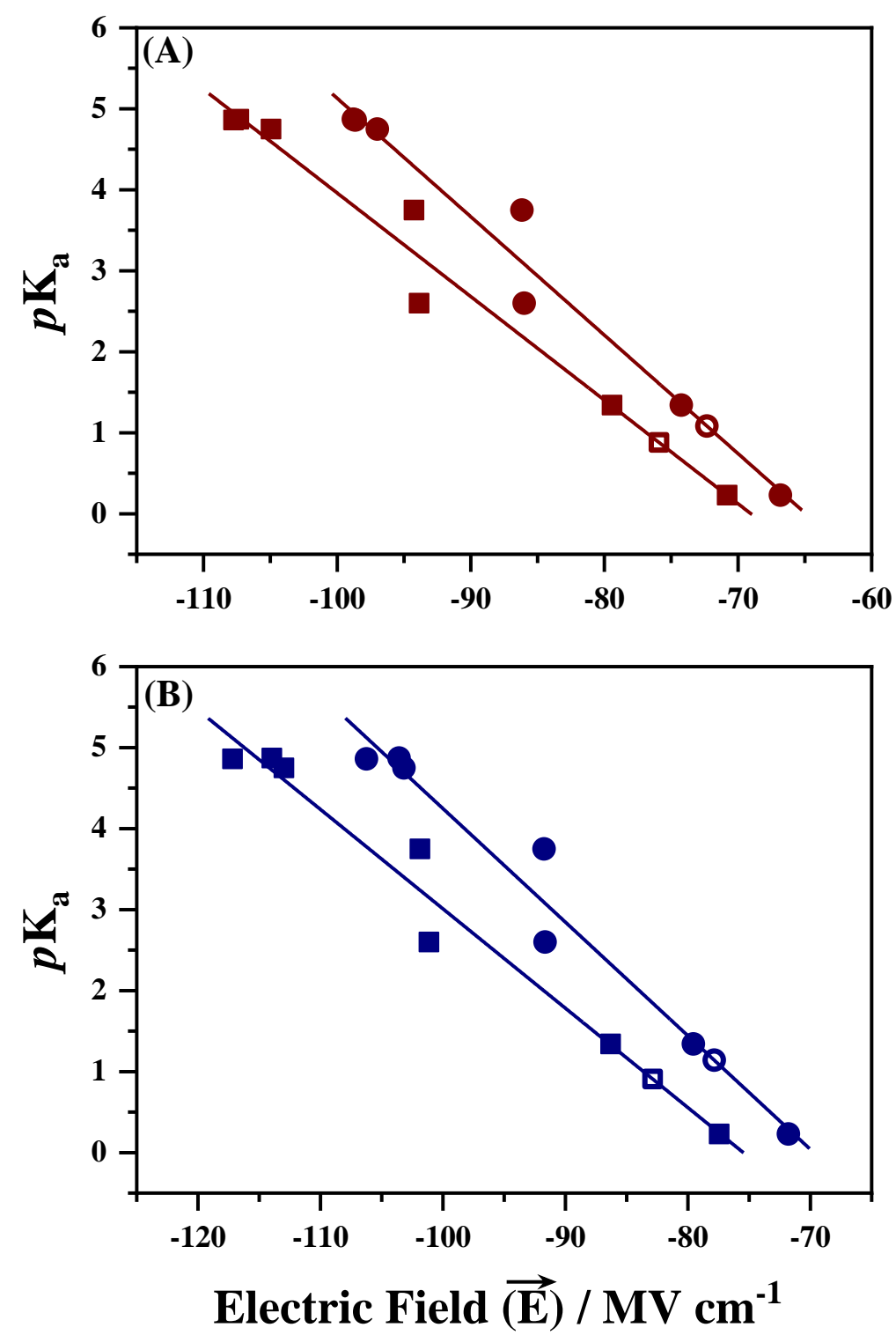

Fig. 5 Plots of $p K_{a}$ of the carboxylic acid against the electric field along the $0-\mathrm{H}$ group (A) acetic acid and (B) trifluoroacetic acid. The electric field data was generated from the corresponding homo/hetero dimers. In each case, the squares, and circles represent the electric field values calculated using MP2 and B3LYP-D3 levels, respectively. The straightlines are linear least-square fits to the data points. In each case, the data point representing open square / open circle is due to fluoroformic acid, whose $p K_{a}$ is unknown and is estimated using the linear correlation. 
The role of electric fields along the $\mathrm{OH}$ groups were further explored through acid dissociation constant $\left(p \mathrm{~K}_{\mathrm{a}}\right)$. Fig. 5 shows the plot of experimental $p \mathrm{~K}_{\mathrm{a}}{ }^{35}$ of the partnering carboxylic acid against the electric field experienced by the $\mathrm{OH}$ group of a particular carboxylic acid in the dimer. For instance, in Fig. $5 \mathrm{~A}$, the $p \mathrm{~K}_{a}$ values of various carboxylic acid are plotted against the field experienced by the $\mathrm{OH}$ group of acetic acid in various homo and hetero dimers of acetic acid. A similar plot for trifluoroacetic acid is shown in Fig. 5B and the remaining six carboxylic acids are shown in Fig. S4 (see the SI). Among the eight carboxylic acids considered in the present work, very little is known about fluoroformic acid as it is known to autocatalytically decompose into $\mathrm{CO}_{2}$ and HF. However, the ionized and protonated forms of fluoroformic acid have been reported to exist in the gas phase. ${ }^{36}$ Moreover, the crystal structure of the anhydrous form of fluoroformic acid has also been reported. ${ }^{37}$ Therefore, based on the linear correlation between the $p \mathrm{~K}_{\mathrm{a}}$ and the electric field along the $\mathrm{OH}$ group in the carboxylic acid dimer (see Figs 6 and S3) the $p \mathrm{Ka}_{\mathrm{a}}$ of fluoroformic acid was estimated to be 0.83 and 1.09 at MP2 and B3LYP-D3 levels of theory, respectively (Table S9, see the SI). In general, the electric field estimated by B3LYP-D3 was lower by about 10\% in comparison to the MP2 level, which is reflected in the higher value of $p \mathrm{~K}_{\mathrm{a}}$. Based on the present set of results, the $p \mathrm{~K}_{\mathrm{a}}$ of fluoroformic acid would lie between the $p \mathrm{~K}_{\mathrm{a}}$ values of difluoroacetic acid (1.34) and trifluoroacetic acid (0.23) and can be estimated to be around 0.9 .

\section{Conclusions}

The electric field experienced by the $\mathrm{OH}$ group in several homo- and hetero- dimers of carboxylic acids were evaluated using MP2 and B3LYP-D3 levels of theory using aug-cc-pVDZ basis set. The $\mathrm{O}-\mathrm{H}$ oscillators in the carboxylic acid dimers are strongly coupled and were decoupled by selective deuteration. The shifts in the $\mathrm{O}-\mathrm{H}$ stretching frequencies were linearly correlated with the electric field yielding the Stark tuning rate $(\Delta \vec{\mu})$ of about 21.9 and $5.7 \mathrm{~cm}^{-1}$ $\left.[\mathrm{MV} \mathrm{cm}]^{-1}\right]^{-1}$ for the primary and secondary substitution effects. The ratio of O-H/O-D Stark tuning rates was found to be around $\sqrt{2}$ which is due to the kinematic effect. The Stark tuning rate for the anharmonic 0-D frequency shifts on average was about $40-50 \%$ higher compared to the corresponding harmonic $0-\mathrm{D}$ frequency shifts, which suggests the presence of strong anharmonicity of O-H/O-D oscillators in carboxylic acid dimers. Based on the linear correlation 
observed between $p \mathrm{~K}_{\mathrm{a}}$ and the electric field, the $p \mathrm{~K}_{\mathrm{a}}$ of fluoroformic acid was estimated to be around 0.9 .

\section{Conflicts of interest}

There are no conflicts to declare

\section{Acknowledgments}

The authors gratefully acknowledge the SpaceTime-2 supercomputing facility at IIT Bombay for the computing time.

\section{References}

1 O. Ivasenko and D. F. Perepichka, Mastering fundamentals of supramolecular design with carboxylic acids. Common lessons from X-ray crystallography and scanning tunneling microscopy, Chem. Soc. Rev., 2011, 40, 191-206.

2 Y. Maréchal, IR spectra of carboxylic acids in the gas phase: A quantitative reinvestigation, J. Chem. Phys., 1987, 87, 6344-6353.

3 K. G. Goroya, Y. Zhu, P. Sun and C. Duan, High resolution jet-cooled infrared absorption spectra of the formic acid dimer: A reinvestigation of the C-O stretch region, J. Chem. Phys., 2014, 140, 164311.

4 G. M. Florio, T. S. Zwier, E. M. Myshakin, K. D. Jordan, E. L. Sibert, G. M. Florio and T. S. Zwier, Theoretical modeling of the $\mathrm{OH}$ stretch infrared spectrum of carboxylic acid dimers based on first-principles anharmonic couplings Theoretical modeling of the $\mathrm{OH}$ stretch infrared spectrum of carboxylic acid dimers based on first-principles anharmonic coupl, 2003, 1735-1746.

5 C. K. Nandi, M. K. Hazra and T. Chakraborty, Vibrational coupling in carboxylic acid dimers, J. Chem. Phys., 2005, 123, 124310.

6 M. D. Elkin, V. V. Smirnov, A. M. Likhter and O. N. Grechuhina, Theoretical Interpretation of the Vibrational Spectra of Carboxylic-Acid Dimers in the High-Frequency Range, J. Surf. Investig. X-ray, Synchrotron Neutron Tech., 2018, 12, 38-43.

7 T. Elsaesser, N. Huse, J. Dreyer, J. R. Dwyer, K. Heyne and E. T. J. J. Nibbering, Ultrafast vibrational dynamics and anharmonic couplings of hydrogen-bonded dimers in solution, Chem. Phys., 2007, 341, 175-188.

8 K. A. E. Meyer and M. A. Suhm, Vibrational exciton coupling in homo and hetero dimers of 
carboxylic acids studied by linear infrared and Raman jet spectroscopy, J. Chem. Phys., 2018, 149, 104307.

9 A. Nejad and M. A. Suhm, Concerted Pair Motion Due to Double Hydrogen Bonding: The Formic Acid Dimer Case, J. Indian Inst. Sci., 2020, 100, 5-19.

10 A. M. Stingel, C. Calabrese and P. B. Petersen, Strong intermolecular vibrational coupling through cyclic hydrogen-bonded structures revealed by ultrafast continuum mid-IR spectroscopy, J. Phys. Chem. B, 2013, 117, 15714-15719.

11 K. B. Beć, Y. Futami, M. J. Wójcik, T. Nakajima and Y. Ozaki, Spectroscopic and Computational Study of Acetic Acid and Its Cyclic Dimer in the Near-Infrared Region, J. Phys. Chem. A, 2016, 120, 6170-6183.

12 C. Emmeluth, M. A. Suhm, D. Luckhaus, C. Emmeluth, M. A. Suhm and D. Luckhaus, A monomers-in-dimers model for carboxylic acid dimers A monomers-in-dimers model for carboxylic acid dimers, 2003, 118, 2242-2255.

13 F. Madeja and M. Havenith, High resolution spectroscopy of carboxylic acid in the gas phase: Observation of proton transfer in (DCOOH)2, J. Chem. Phys., 2002, 117, 7162-7168.

14 C. S. Tautermann, A. F. Voegele and K. R. Liedl, The ground-state tunneling splitting of various carboxylic acid dimers, J. Chem. Phys., 2004, 120, 631-637.

15 T. Loerting and K. R. Liedl, Toward elimination of discrepancies between theory and experiment: Double proton transfer in dimers of carboxylic acids, J. Am. Chem. Soc., 1998, 120, 12595-12600.

16 W. Li, L. Evangelisti, Q. Gou, W. Caminati and R. Meyer, The Barrier to Proton Transfer in the Dimer of Formic Acid: A Pure Rotational Study, Angew. Chemie - Int. Ed., 2019, 58, 859-865.

17 K. Mackeprang, Z. H. Xu, Z. Maroun, M. Meuwly and H. G. Kjaergaard, Spectroscopy and dynamics of double proton transfer in formic acid dimer, Phys. Chem. Chem. Phys., 2016, 18, 24654-24662.

18 L. Evangelisti, P. Écija, E. J. Cocinero, F. Castaño, A. Lesarri, W. Caminati and R. Meyer, Proton tunneling in heterodimers of carboxylic acids: A rotational study of the benzoic acid-formic acid bimolecule, J. Phys. Chem. Lett., 2012, 3, 3770-3775.

19 M. Katada and A. Fujii, Infrared spectroscopic evidence for the initial step of dissociation of the stable benzoic acid cyclic dimer with microsolvation by a single water molecule, Chem. Phys. Lett., 2017, 684, 368-372.

20 P. Krishnakumar and D. K. Maity, Theoretical studies on dimerization vs . microhydration of carboxylic acids, Comput. Theor. Chem., 2017, 1099, 185-194.

21 Y. C. Hu, X. H. Zhang, Q. S. Li, Y. H. Zhang and Z. S. Li, Effect of Water on the Structure and Stability of Hydrogen-Bonded Oxalic Acid Dimer, ChemPhysChem, 2017, 18, 3375-3383.

22 I. Tsivintzelis, G. M. Kontogeorgis and C. Panayiotou, Dimerization of Carboxylic Acids: An Equation of State Approach, J. Phys. Chem. B, 2017, 121, 2153-2163.

23 B. L. Van Hoozen, P. B. Petersen, B. L. Van Hoozen and P. B. Petersen, Vibrational tug-ofwar : The pKA dependence of the broad vibrational features of strongly hydrogen-bonded 
carboxylic acids Vibrational tug-of-war: The pK A dependence of the broad vibrational features of strongly hydrogen-bonded carboxylic acids, 2018, 148, 134309.

24 C. O. Da Silva, E. C. Da Silva and M. A. C. Nascimento, Ab initio calculations of absolute pKa values in aqueous solution I. Carboxylic acids, J. Phys. Chem. A, 1999, 103, 11194-11199.

25 C. O. Silva, E. C. Da Silva and M. A. C. Nascimento, Ab initio calculations of absolute pKa values in aqueous solution II. Aliphatic alcohol, thiols, and halogenated carboxylic acids, J. Phys. Chem. A, 2000, 104, 2402-2409.

26 M. Namazian and S. Halvani, Calculations of pKa values of carboxylic acids in aqueous solution using density functional theory, J. Chem. Thermodyn., 2006, 38, 1495-1502.

27 M. D. Liptak and G. C. Shields, Accurate pKa calculations for carboxylic acids using Complete Basis Set and Gaussian-n models combined with CPCM continuum solvation methods, J. Am. Chem. Soc., 2001, 123, 7314-7319.

28 S. Sen, M. Boda, S. Venkat Lata and G. Naresh Patwari, Internal electric fields in small water clusters [(H 2 O) n ; n = 2-6], Phys. Chem. Chem. Phys., 2016, 18, 16730-16737.

29 M. Boda and G. Naresh Patwari, Insights into acid dissociation of $\mathrm{HCl}$ and $\mathrm{HBr}$ with internal electric fields, Phys. Chem. Chem. Phys., 2017, 19, 7461-7464.

30 S. D. Fried and S. G. Boxer, Measuring electric fields and noncovalent interactions using the vibrational stark effect, Acc. Chem. Res., 2015, 48, 998-1006.

31 M. Saggu, N. M. Levinson and S. G. Boxer, Direct measurements of electric fields in weak OH $\cdots \pi$ hydrogen bonds, J. Am. Chem. Soc., 2011, 133, 17414-17419.

32 M. Saggu, N. M. Levinson and S. G. Boxer, Experimental quantification of electrostatics in $\mathrm{X}-\mathrm{H} \cdots \varphi$ hydrogen bonds, J. Am. Chem. Soc., 2012, 134, 18986-18997.

33 S. R. Gadre and R. N. Shirsat, Electrostatics of atoms and molecules, University Press, Hyderabad, 2000.

34 M. J. Frisch, G. W. Trucks, H. B. Schlegel, G. E. Scuseria, M. A. Robb, J. R. Cheeseman, G. Scalmani, V. Barone, G. A. Petersson, H. Nakatsuji, X. Li, M. Caricato, A. V. Marenich, J. Bloino, B. G. Janesko, R. Gomperts, B. Mennucci, H. P. Hratchian, J. V. Ortiz, A. F. Izmaylov, J. L. Sonnenberg, D. Williams-Young, F. Ding, F. Lipparini, F. Egidi, J. Goings, B. Peng, A. Petrone, T. Henderson, D. Ranasinghe, V. G. Zakrzewski, J. Gao, N. Rega, G. Zheng, W. Liang, M. Hada, M. Ehara, K. Toyota, R. Fukuda, J. Hasegawa, M. Ishida, T. Nakajima, Y. Honda, 0. Kitao, H. Nakai, T. Vreven, K. Throssell, J. Montgomery, J. A., J. E. Peralta, F. Ogliaro, M. J. Bearpark, J. J. Heyd, E. N. Brothers, K. N. Kudin, V. N. Staroverov, T. A. Keith, R. Kobayashi, J. Normand, K. Raghavachari, A. P. Rendell, J. C. Burant, S. S. Iyengar, J. Tomasi, M. Cossi, J. M. Millam, M. Klene, C. Adamo, R. Cammi, J. W. Ochterski, R. L. Martin, K. Morokuma, O. Farkas, J. B. Foresman and D. J. Fox, Gaussian-16, Rev. B.01, Gaussian, Inc., Wallingford CT, 2016.

35 M. Boda and G. N. Patwari, Internal electric fields in methanol [MeOH]2-6 clusters, Phys. Chem. Chem. Phys., 2020, 22, 10917-10923.

36 D. Sadhukhan, A. Hazra and G. Naresh Patwari, Bend-to-Break: Curvilinear Proton Transfer in Phenol-Ammonia Clusters, J Phys Chem A, 2020, 124, 3101-3108. 